\section{Is HIV the causative factor in AIDS?}

SIR-As a participant at the forum in which Peter Duesberg faced his critics (Nature 332, 574; 1988), it is my opinion that the Byzantine symptomatology of AIDS is unlikely to be simply accounted for by immunodeficiency supposedly caused by the retrovirus termed HIV.

This is based on experience with the natural spread of another retrovirus, avian leukosis virus (ALV), assumed since 1910 to be responsible for visceral lymphomatosis (VL) in chickens $(\mathrm{H}$. Rubin et al. Virology 17, 143; 1962). I found that $25 \%$ of about 600 chickens in a susceptible flock were congenitally infected with ALV, and almost every cell actively produced virus throughout a bird's lifetime. The other birds became infected by contact after hatching and developed antibodies.

Despite massive and continuous active infection of the cogenitally infected chickens, their growth and appearance was the same as that of the other birds. Only $15 \%$ of the congenitally infected birds developed VL and only $3 \%$ of the contact-infected birds, about the same percentage that develop $\mathrm{VL}$ in the absence of ALV infection. Although the virus may play a role in accelerating the onset of the disease, clearly there are other factors involved in its occurrence, including life history and genetic makeup. In an ordinary commercial flock only $3 \%$ were congenitally infected, with most of the rest becoming infected by contact. If the occurrence of VL in the infected population of a commercial flock is as low as that in our experimental flock, the overall occurrence of VL would be no higher than in an uninfected flock and the role of the virus, in the absence of other factors, would be moot.

I have found no convincing evidence in the AIDS literature or at the forum that HIV is more cytopathic in vivo than ALV. Even were HIV more cytopathic, it is unlikely to account directly for the immune deficiency in view of the tiny fraction, less than 1 in 10,000 , of lymphocytes actively infected. The protagonists of HIV causation of AIDS at the forum discounted the significance of other factors prevalent in the high-risk groups, such as multiple infections with other viruses and bacteria, and the debilitating effects on the immune system of repeated receptive anal intercourse, intravenous drug abuse and heavy antibiotic consumption. These practices have greatly intensified in the United States with the advent of gay liberation and the drug epidemic.

The annual AIDS morbidity of $5 \%$ among those with HIV antibody in highrisk groups predicts 50,000 to 75,000 new cases per year among the 1-1.5 million US antibody positives, which is 3-5 times higher than the actual annual occurrence. In fact, it is possible that AIDS morbidity is very low in the absence of 'cofactors', and might approach zero in the healthy heterosexual population, as Duesberg claims. In a complex syndrome like AIDS it is difficult indeed to disentangle cause from correlation without much more data on disease incidence among HIV-positive individuals in low-risk groups. The usual counter argument to any question of the primeval role of HIV in AIDS is the citation of cases induced by transfusion; but this has to be considered alongside the absence of the disease in over 750 accidental needle sticks with AIDS-contaminated blood. In short, the picture is far from simple and it is a disservice to base our health practices solely on that picture.

HARRY RUBIN

Department of Molecular Biology,

University of California, Berkeley,

California 94720 , USA

\section{Fossil carbon sources of atmospheric methane}

SIR-In support of a recent report ${ }^{1}$ that about $32 \%$ of atmospheric methane is derived from fossil carbon, we would like to suggest two petroleum-related sources that have not received sufficient attention.

The first is the underwater venting of waste or non-commercial quantities of natural gas from offshore production platforms - estimated to be responsible for up to $70 \%$ of the gases being flared or vented offshore in the US ${ }^{2}$. Underwater venting was common in the 1970 s in the outer continental-shelf (OCS) region of the US, amounting to a few per cent of the total gas produced. Sea Technology ${ }^{3}$ gives a figure of 60 million MCF $(1,000$ cubic feet) in the OCS region in 1973. One may thus calculate that about $10^{12} \mathrm{~g}$ of methane were released to the atmosphere by this practice in this region in 1973. Considering the looser production practices in other parts of the world, for example, the Persian Gulf, underwater venting may be contributing several teragrams $\left(10^{12} \mathrm{~g}=1\right.$ $\mathrm{Tg}$ ) of methane per year to the fossil component of the atmospheric methane source budget.

The second source of petroleum production-related methane is that photochemically produced by sunlight on asphalts. As much as 678,000 barrels per day of asphalt were produced in the US in $1984^{4}$. This may be converted to $3.28 \times$ $10^{13} \mathrm{~g}$ carbon per year. In the laboratory we can get about $15 \%$ conversion of similar materials to methane by thermal processes. Also we can show that the Sun's ultraviolet radiation can produce methane from asphalt. Using the estimates given above, potentially $5 \mathrm{Tg}$ of $\mathrm{CH}_{4}$ per year could be formed from newly produced asphalt by photochemical and thermal induced processes. Considering worldwide asphalt production, its methane producing potential is even larger. Asphalt has a long lifetime in the environment. It is used extensively for road surfaces and roofing, both excellently exposed to the Sun's radiant energy.

We recommend that these sources of fossil methane be thoroughly evaluated in future studies of methane budgets.

WILliam M. SACKETT

TIMOTHY R. BARBER

Department of Marine Science,

University of South Florida,

Florida 33701, USA

1. Lowe, D. C., et al. Nature 332, 522-525 (1988).

2. Brooks, J. M. Bernard, B. B. \& Sackett, W. M., in Fate and Effects of Hydrocarbons in Marine Ecosystems and Organ isms (ed. Wolfe, D. A.) 373 (1977)

Sea Technol 16, 10 (1974)

4. Cantrell, A. Oil Gas J. 32, 111 (1984).

\section{Sexual selection in guppies defended}

SIR-In a second criticism ${ }^{1}$ of our finding ${ }^{2}$ that predation risk determines female mating preferences in wild guppies, Houde restates Endler's points ${ }^{3}$, which we have previously answered ${ }^{4}$, and raises new objections. But these could be raised against most studies in evolutionary biology, which can at best include only a small number of populations and cannot positively preclude all alternative explanations.

First, Houde objects that we studied only two high-predation and two lowpredation populations. But a strength of our investigation is that we examined more than one population, compared to previous studies of female preference, and we are presently examining more populations from a wider range of environmental conditions.

Second, Houde states that female preference must be studied under a wider range of experimental conditions, such as open versus partitioned aquaria. We agree, and in our earlier reply to Endler ${ }^{4}$ we suggested how our system using models of male guppies is ideally suited to provide such information.

Finally, Houde suggests that more information on selection in natural populations is necessary. In a companion article $^{5}$ on variation in female preferences tested with live males, published in a format allowing a more expansive discussion, we have listed the types of data on selection that are needed for a full understanding of the evolution of female preference in this species. These include the determination of whether direct selection acts on females, whether the optimum male phenotype, the intensity of selection around the optimum, or both factors 